\title{
Influence of Specimen Preparation on SEM Images and Comparison to LOM Images
}

George F. Vander Voort, Consultant, Struers Inc.

SEM and LOM are sometimes viewed as competitive methods; but, they are actually complementary. Some people believe that specimen preparation is less critical for SEM work than for LOM work; but, the reverse is true. Image forming mechanisms for the LOM and the SEM are different - we should make the most of these differences. The LOM is a more efficient tool for fast, low magnification $(\leq 2000 \mathrm{X})$ examination of a specimen - use it first to determine if, and where, one should follow with higher magnification SEM examination and EDS or EBSD work.

Prepare every specimen for SEM examination with the quality required for EBSD work; this is actually quite easy with the right equipment, consumables and methods. Examples of preparation methods for many metals/alloys and other materials can be found in Struers publications and web site. Examples of LOM and SEM images of specimens that were properly prepared or poorly prepared will be shown.

First, specimens must be properly prepared to a very high quality level. As with EBSD, sectioning must induce minimal damage. Use abrasive cut-off machines with a blade/wheel designed for metallography and for the material being cut. Grind with the best surface for the material being prepared that will induce the least damage while removing cutting damage in a reasonable time.

Next, polish the specimens using flat low resilience cloths, such as DP/MD Plan or DP/MDPan with 9- $\mu \mathrm{m}$ diamond, using a load of 25-30 N per specimen, $150 \mathrm{rpm}$, for at least 5 minutes. Next, polish with DP/MD DAC, DP/MD DUR or DP/MD SAT cloths with 3- $\mu \mathrm{m}$ diamond, same load and rpm, for 5 minutes. For some materials, a 1- $\mu \mathrm{m}$ step may be necessary, for 3-5 minutes using the same cloths and conditions as for 3- $\mu \mathrm{m}$ diamond. The final step would be performed using either a synthetic neoprene cloth, such as DP/MDChem, or a napped or flocked cloth, such as DP/MD-Floc or DP/MD-Nap using either colloidal silica, such as OP-S or a neutral alumina suspension, such as OP-AN. Polishing is usually conducted at 120-150 rpm, same load, from 1 to 3 minutes. A good practice is to lightly etch the specimens after the last step with a general-purpose reagent to see what the structure actually is and determine how well prepared the specimens are before proceeding to examination. After examination, repeat the last step for at least 1 minute to remove this etch. Cleaning after each step is important to prevent contamination of the next step and poor results.

If the material has phases or constituents with strong atomic number contrast, etching is not needed for SEM examination with BSE. Likewise, for many single-phase microstructures, 
as-polished specimens will exhibit a grain-contrast luster when examined with BSE. For LOM examination, un-etched non-cubic crystal structure metals/alloys can be examined more effectively in polarized light when properly prepared than if etched and examined with bright field illumination. Figure1 illustrates the quality difference in images properly prepared vs less well prepared.

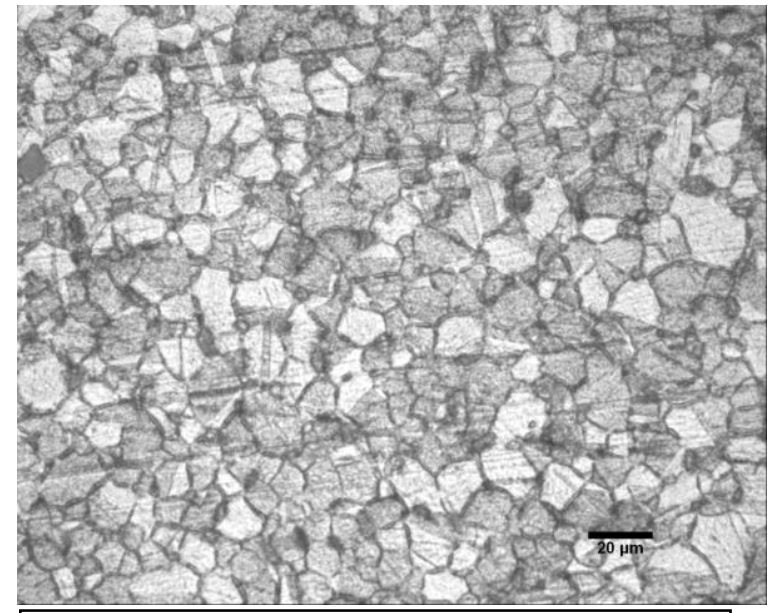

Equal Parts $3 \% \mathrm{H}_{2} \mathrm{O}_{2}$ and $\mathrm{NH}_{4} \mathrm{OH}$

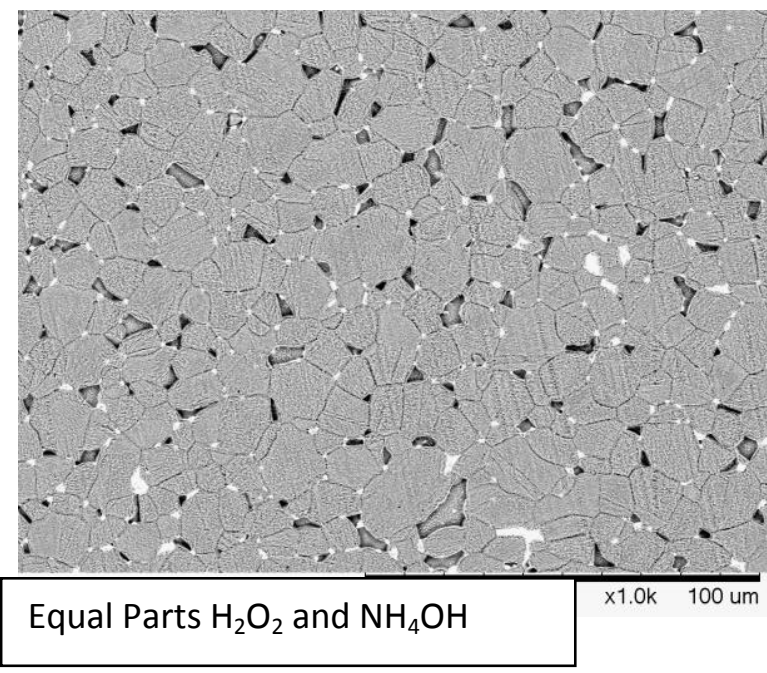

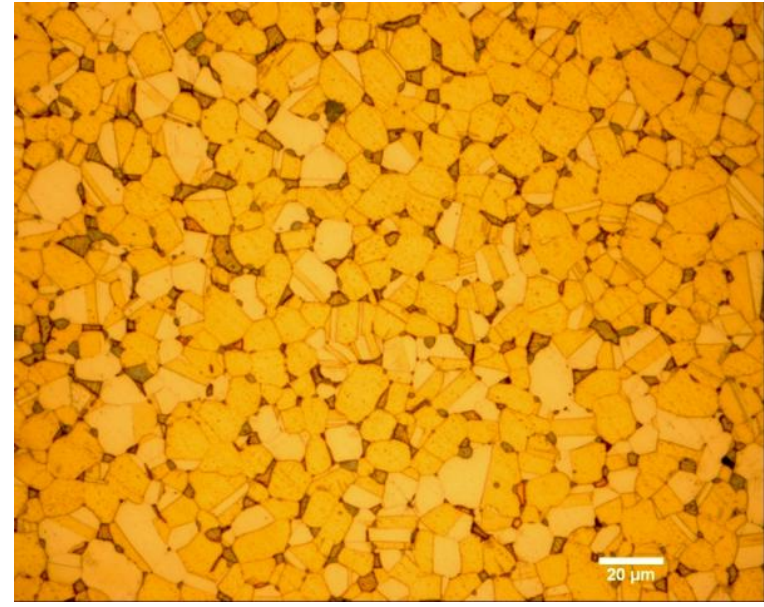

Klemm's I

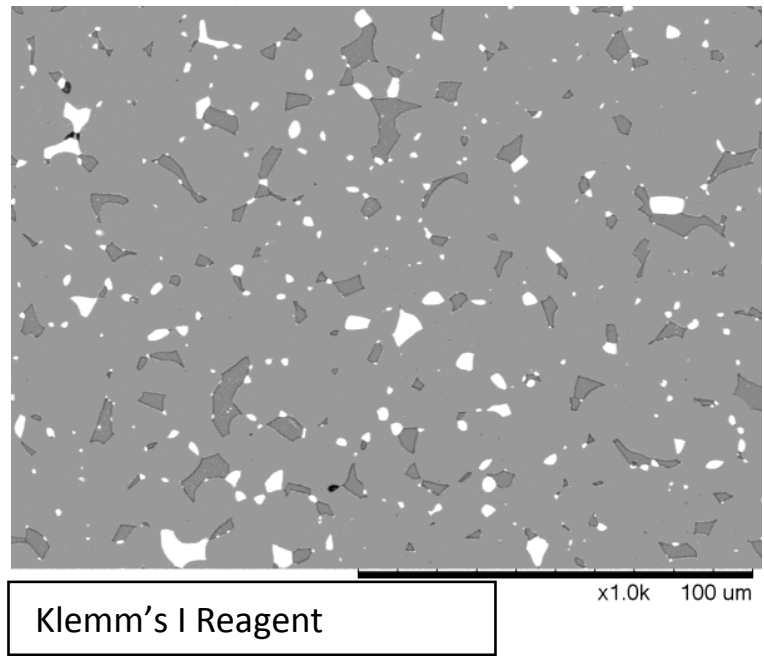

Figure 1. LOM (top at 500X) and SEM BSE (bottom at 1000X) images of poorly prepared (left) and properly prepared (right) specimens of leaded brass $(\mathrm{Cu}-30 \% \mathrm{Zn}-3 \% \mathrm{~Pb})$. Note the distinct difference in image quality between the well-prepared specimens compared to the more poorly prepared specimens. 